\title{
THE ERGODIC HILBERT TRANSFORM FOR CESĀRO BOUNDED FLOWS
}

\author{
María Lorente Domínguez and Francisco Javier Martín-Reyes
}

(Received August 6, 1993, revised February 14, 1994)

\begin{abstract}
The aim of this paper is to study the behaviour of the ergodic Hilbert transform associated to a flow which is Cesàro bounded in the space of integrable functions. In particular we see that if the flow is Cesàro bounded in this space, and $f$ and its ergodic Hilbert transform are integrable functions then the ergodic Hilbert transform is not only defined as an a.e. limit or a limit in measure, but it is also defined as a limit in the norm of the space of integrable functions. In order to prove this result, we show that the ergodic Hilbert transform and the ergodic maximal operator are of weak type $(1,1)$ if the flow is Cesàro bounded in the space of integrable functions. It is also shown that the ergodic Hilbert transform and the ergodic maximal operator are of strong type $(p, p)$, with $p$ greater than one and finite, if the flow is Cesàro bounded in the space of measurable functions with integrable $p$ th-power. The last section of the paper is devoted to providing nontrivial examples of Cesàro bounded flows. The proofs use ideas of the theory of Muckenhoupt's weights.
\end{abstract}

1. Introduction. Let $(X, \mathscr{M}, v)$ be a finite measure space. By a flow $\left\{\tau_{t}: t \in \boldsymbol{R}\right\}$ we mean a group of measurable transformations $\tau_{t}: X \rightarrow X$ with $\tau_{0}$ the identity and $\tau_{t+s}=\tau_{t} \circ \tau_{s},(t, s \in R)$. The flow is said to be measure preserving if the $\tau_{t}$ are measure preserving, i.e., if $v\left(\tau_{-t} E\right)=v(E)$ for all $E \in \mathscr{M}$. The flow is said to be nonsingular if $v\left(\tau_{-t} E\right)=0$ for all $t \in \boldsymbol{R}$ and all $E \in \mathscr{M}$ with $v(E)=0$. Finally, the flow is said to be measurable if the $\operatorname{map}(x, t) \rightarrow \tau_{t} x$ from $X \times \boldsymbol{R}$ into $X$ is $\tilde{\mathscr{M}}$ - $\mathscr{M}$-measurable where $\tilde{\mathscr{M}}$ is the completion of the product- $\sigma$-algebra $\mathscr{M} \otimes \mathscr{B}$ of $\mathscr{M}$ with the Borel sets, and the completion is taken with respect to the product measure of $v$ on $\mathscr{M}$ and the Lebesgue measure on $\mathscr{B}$.

Let $f: X \rightarrow \boldsymbol{R}$ be a measurable function. The ergodic averages $A_{\varepsilon} f, \varepsilon>0$, and the ergodic maximal operator $M f$ associated to a measurable flow are defined by

$$
A_{\varepsilon} f(x)=\frac{1}{2 \varepsilon} \int_{-\varepsilon}^{\varepsilon} f\left(\tau_{t} x\right) d t
$$

and

1991 Mathematics Subject Classification. 28D05.

Key words and phrases. Cesàro bounded flows, ergodic Hilbert transform, ergodic maximal operator, weights.

This research has been partially supported by D.G.I.C.Y.T. grant (PB91-0413) and Junta de Andalucía. 


$$
M f(x)=\sup _{\varepsilon>0} A_{\varepsilon}|f|(x) .
$$

We also consider the maximal ergodic Hilbert transform

$$
H^{*} f(x)=\sup _{\varepsilon>0}\left|H_{\varepsilon} f(x)\right|
$$

where

$$
H_{\varepsilon} f(x)=\int_{\varepsilon<|t|<1 / \varepsilon} \frac{f\left(\tau_{t} x\right)}{t} d t .
$$

Assume now that the measurable flow $\left\{\tau_{t}: t \in \boldsymbol{R}\right\}$ is measure preserving. Then $M$ and $H^{*}$ are of weak type $(1,1)$ and of strong type $(p, p), 1<p<\infty$, i.e., the following inequalities hold, where $\mathscr{R}$ is either $M$ or $H^{*}$ :

$$
v(\{x \in X:|\mathscr{R} f(x)|>\lambda\}) \leq \frac{C}{\lambda} \int_{X}|f| d v
$$

and

$$
\int_{X}|\mathscr{R} f|^{p} d v \leq C \int_{X}|f|^{p} d v, \quad 1<p<\infty .
$$

It follows from these results that $A_{\varepsilon} f$ converges a.e. as $\varepsilon$ goes to 0 for all $f \in L^{1}(d v)$. In the same way it is seen that the ergodic Hilbert transform

$$
H f(x)=\lim _{\varepsilon \rightarrow 0} H_{\varepsilon} f(x)
$$

exists a.e. (the limit is understood in the pointwise sense). An immediate consequence of the above inequalities is that if $f \in L^{p}(d v), 1 \leq p<\infty$, then $A_{\varepsilon} f$ converges in the $L^{p_{-}}$ norm. We also have that if $f \in L^{p}(d v), 1<p<\infty$, then $H_{\varepsilon} f$ converges to $H f$ in the $L^{p_{-}}$ norm and if $f \in L^{1}(d v)$ then $H_{\varepsilon} f$ converges to $H f$ in measure (see [P] for these results). This leaves open the following question: if $f \in L^{1}(d v)$ and $H f \in L^{1}(d v)$ does $H_{\varepsilon} f$ converge to $H f$ in the $L^{1}$-norm as $\varepsilon$ goes to 0 ? This question was answered in the affirmative in $[A B G]$ (in fact the setting of this paper is about power-bounded operators not just measure preserving flows).

Our aim is to study the behaviour of the ergodic Hilbert transform associated to a nonsingular measurable flow which need not preserve the measure $v$ but which is a Cesàro-bounded flow, i.e.,

$$
\sup _{\varepsilon>0}\left\|A_{\varepsilon} f\right\|_{L^{p}(d v)} \leq C\|f\|_{L^{p}(d v)}
$$

for some fixed $p, 1 \leq p<\infty$. Observe that this case is not included in [ABG]. The results on $L^{p}(d v), 1<p<\infty$, are easier and their discrete versions can be found in [MT] and 
[S]. We include them because we use them to study the ergodic Hilbert transform in $L^{1}(d v)$. Our main results are collected in the following theorems.

THEOREM 1. Let $1 \leq p<\infty$. Assume that $(X, \mathscr{M}, v)$ is a finite measure space and let $\left\{\tau_{t}: t \in \boldsymbol{R}\right\}$ be a nonsingular measurable flow on $X$ such that

$$
\sup _{\varepsilon>0}\left\|A_{\varepsilon} f\right\|_{L^{p}(d v)} \leq C\|f\|_{L^{p}(d v)}
$$

for some positive constant $C$ and all $f \in L^{p}(d v)$. Let us denote by $\mathscr{R}$ either $M$ or $H^{*}$.

(a) If $p=1$ then there exists $C>0$ such that

$$
v(\{x \in X:|\mathscr{R} f(x)|>\lambda\}) \leq \frac{C}{\lambda} \int_{X}|f| d v
$$

for all $\lambda>0$ and all functions $f \in L^{1}(d v)$.

(b) If $p>1$ then there exists $C>0$ such that

$$
\int_{X}|\mathscr{R} f|^{p} d v \leq C \int_{X}|f|^{p} d v
$$

for all functions $f \in L^{p}(d v)$.

(c) For every $f \in L^{p}(d v)$, the limits of $A_{\varepsilon} f(x)$ as $\varepsilon$ goes to $\infty$ and of $H_{\varepsilon} f(x)$ as $\varepsilon$ goes to 0 exist a.e.

A straightforward consequence of Theorem 1 is the following:

COROLlaRY 1. Under the same assumptions as that in Theorem 1, we have:

(a) If $1<p<\infty$ and $f \in L^{p}(d v)$ then $A_{\varepsilon} f$ converges in the $L^{p}(d v)$-norm as $\varepsilon$ goes to $\infty$ and $H_{\varepsilon} f$ converges in the $L^{p}(d v)$-norm as $\varepsilon$ goes to 0 .

(b) If $p=1$ and $f \in L^{1}(d v)$ then $A_{\varepsilon} f$ converges in the $L^{1}(d v)$-norm as $\varepsilon$ goes to $\infty$ and $H_{\varepsilon} f$ converges in measure as $\varepsilon$ goes to 0 .

THEOREM 2. Assume that $(X, \mathcal{M}, v)$ is a finite measure space and let $\left\{\tau_{t}: t \in \boldsymbol{R}\right\}$ be a nonsingular measurable flow defined on $X$ such that

$$
\sup _{\varepsilon>0}\left\|A_{\varepsilon} f\right\|_{L^{1}(d v)} \leq C\|f\|_{L^{1}(d v)}
$$

for some $C>0$ and all functions $f \in L^{1}(d v)$. If $f \in L^{1}(d v)$ and $H f \in L^{1}(d v)$ then $H_{\varepsilon} f$ converges to $H f$ in the $L^{1}(d v)$-norm as $\varepsilon$ goes to 0 .

The proof of this theorem appears in Sections 4 and 5 and follows the ideas in [ABG], [CC] and [CS]. The latter papers study the behaviour of the (classical) Hilbert transform on the real line. Section 2 will be devoted to some results we shall use about the Hilbert transform and the Hardy-Littlewood maximal operator while the proof of Theorem 1 is in Section 3. Finally, Section 6 gives examples of nontrivial Cesàrobounded flows. 
Throughout this paper, the letter $C$ will always mean a positive constant not necessarily the same at each occurrence and if $1<p<\infty$ then $p^{\prime}$ will denote its conjugate exponent, i.e., the number $p^{\prime}$ such that $p+p^{\prime}=p p^{\prime}$.

2. Results in harmonic analysis. In order to prove the theorems we will need some results on the Hardy-Littlewood maximal operator and the Hilbert transform in $\boldsymbol{R}$. We recall that for a locally integrable function $f$ on the real line, the HardyLittlewood maximal function, the maximal Hilbert transform and the Hilbert transform are defined respectively by

$$
\begin{aligned}
f^{*}(x) & =\sup _{\varepsilon>0} \frac{1}{2 \varepsilon} \int_{-\varepsilon}^{\varepsilon}|f(x-t)| d t, \\
K^{*} f(x) & =\sup _{\varepsilon>0}\left|\int_{\varepsilon<|t|<1 / \varepsilon} \frac{f(x-t)}{t} d t\right|,
\end{aligned}
$$

and

$$
K f(x)=\lim _{\varepsilon \rightarrow 0^{+}} \int_{\varepsilon<|t|<1 / \varepsilon} \frac{f(x-t)}{t} d t
$$

if the limit exists a.e. We observe that they are, respectively, the ergodic maximal operator, the maximal ergodic Hilbert transform and the ergodic Hilbert transform associated to the flow $\tau_{t}(x)=x-t$ on the real line. The next result will be useful in what follows and you could find its proof in $[\mathrm{HMuW}]$ and $[\mathrm{CF}]$ (see also $[\mathrm{GR}]$ ). In order to state the result, we introduce the following definition.

DEFINITION 1. Let $\omega$ be a positive measurable function on the real line. We say that $\omega$ satisfies $A_{p}, 1 \leq p<\infty$, if there exists a constant $C>0$ such that

$$
\sup _{a<b} \frac{1}{b-a} \int_{a}^{b} \omega(t) d t\left(\frac{1}{b-a} \int_{a}^{b} \omega^{1-p^{\prime}}(t) d t\right)^{p-1} \leq C \quad \text { if } \quad 1<p<\infty
$$

and

$$
\omega^{*}(x) \leq C \omega(x) \text { a.e. if } p=1 .
$$

Now we can state the results we shall use.

THEOREM A. Let $\omega$ be a positive measurable function on $\boldsymbol{R}$. Let $1<p<\infty$ and let $\mathscr{R} f$ be either $f^{*}, K^{*} f$ or $K f$. The following are equivalent.

(a) $\omega$ satisfies $A_{p}$.

(b) There exists $C>0$ such that

$$
\int_{-\infty}^{\infty}|\mathscr{R} f|^{p}(t) \omega(t) d t \leq C \int_{-\infty}^{\infty}|f(t)|^{p} \omega(t) d t
$$


for all functions $f \in L^{p}(\omega(t) d t)$.

(c) There exists $C>0$ such that

$$
\int_{\{t:|\mathscr{R} f(t)|>\lambda\}} \omega(t) d t \leq \frac{C}{\lambda^{p}} \int_{-\infty}^{\infty}|f(t)|^{p} \omega(t) d t
$$

for all $\lambda>0$ and all functions $f \in L^{p}(\omega(t) d t)$.

THEOREM B. Let $\omega$ be a positive measurable function and let $\mathscr{R} f$ be either $f^{*}, K^{*} f$ or $K f$. The following are equivalent.

(a) $\omega$ satisfies $A_{1}$.

(b) There exists $C>0$ such that

$$
\int_{\{t:|\mathscr{R} f(t)|>\lambda\}} \omega(t) d t \leq \frac{C}{\lambda} \int_{-\infty}^{\infty}|f(t)| \omega(t) d t
$$

for all $\lambda>0$ and all functions $f \in L^{1}(\omega(t) d t)$.

3. Proof of Theorem 1. In order to prove Theorem 1, we first claim that there exists a measure $\mu$ equivalent to $v$ such that the flow preserves the measure $\mu$, i.e., $\mu(E)=0$ if and only if $v(E)=0$ and $\mu\left(\tau_{t} E\right)=\mu(E)$ for all sets $E \in \mathscr{M}$.

Proof of The Claim. Since $v(X)<\infty$ we have

$$
\sup _{\varepsilon>0}\left\|A_{\varepsilon} f\right\|_{L^{1}(d v)} \leq C\|f\|_{L^{p}(d v)}(v(X))^{1 / p^{\prime}}<\infty .
$$

Thus the sequence $\left\{\int_{X} A_{n} \chi_{E} d v\right\}_{n}$ is bounded for all $E \in \mathscr{M}$. Let $L$ be a Banach limit. We define

$$
\mu(E)=L\left(\left\{\int_{X} A_{n} \chi_{E} d v\right\}_{n}\right) .
$$

It is clear that $\mu$ is finitely additive and $\mu(\varnothing)=0$. Furthermore, we get from the above inequality

$$
0 \leq \mu(E) \leq C(v(X))^{1 / p^{\prime}}(v(E))^{1 / p} \leq C v(X)<\infty
$$

and then $\mu(X)<\infty$. Therefore, in order to prove that $\mu$ is a countably additive measure, we only have to show that if $E_{1} \supset E_{2} \supset \cdots \supset E_{n} \supset E_{n+1} \supset \cdots$ and $\bigcap_{n} E_{n}=\varnothing, E_{n} \in \mathscr{M}$, then $\mu\left(E_{n}\right) \rightarrow 0$, but this follows from $\mu(E) \leq C(v(X))^{1 / p^{\prime}}(v(E))^{1 / p}$ and the fact that $v$ is a measure.

Now we will see that $\mu\left(\tau_{t} E\right)=\mu(E)$ for all sets $E \in \mathscr{M}$. By the definition of $\mu$ we have

$$
\mu\left(\tau_{t} E\right)=L\left(\left\{\frac{1}{2 n} \int_{-n}^{n} v\left(\tau_{s+t} E\right) d s\right\}_{n}\right) .
$$

Observe that 


$$
\lim _{n \rightarrow \infty}\left[\frac{1}{2 n} \int_{-n}^{n} v\left(\tau_{s+t} E\right) d s-\frac{1}{2 n} \int_{-n}^{n} v\left(\tau_{s} E\right) d s\right]=0 .
$$

In fact,

$$
\left|\frac{1}{2 n} \int_{-n}^{n}\left(v\left(\tau_{s+t} E\right)-v\left(\tau_{s} E\right)\right) d s\right| \leq \frac{t}{n}\left\|A_{t} \chi_{\tau_{n} E}\right\|_{L^{1}(d v)}+\frac{t}{n}\left\|A_{t} \chi_{\tau_{-n} E}\right\|_{L^{1}(d v)} \leq C \frac{t}{n} v(X) .
$$

Therefore, by the properties of the Banach limit

$$
\mu\left(\tau_{t} E\right)=L\left(\left\{\frac{1}{2 n} \int_{-n}^{n} v\left(\tau_{s} E\right) d s\right\}_{n}\right)=\mu(E) .
$$

The proof of the claim will be complete if we show that $v$ and $\mu$ are equivalent. It is obvious that $v(E)=0$ implies $\mu(E)=0$. Assume now that $\mu(E)=0$. Let $B=\bigcup_{k=-\infty}^{\infty} \tau_{k} E$ and let us define

$$
\tilde{v}(E)=\int_{0}^{1} v\left(\tau_{s} E\right) d s
$$

It is clear that $\mu(B)=0$ and

$$
\mu(E)=L\left(\left\{\frac{1}{2 n} \sum_{k=-n}^{n-1} \tilde{v}\left(\tau_{k} E\right)\right\}_{n}\right) .
$$

If we apply this equality to $B$ and keep in mind that $B=\tau_{k} B$ for all integers $k$ then we obtain

$$
0=\mu(B)=L(\{\tilde{v}(B)\})=\tilde{v}(B) .
$$

Thus $v\left(\tau_{s} B\right)=0$ a.e. $s \in(0,1)$, but this implies $v(B)=0$ and then $v(E)=0$.

In what follows, $\omega$ will be the Radon-Nikodym derivative of $v$ with respect to $\mu$. It is clear that $0<\omega<\infty$ a.e.

Proof of Theorem 1. Assume that $p=1$. Observe that

$$
\sup _{\varepsilon>0}\left\|A_{\varepsilon} f\right\|_{L^{1}(d v)} \leq C\|f\|_{L^{1}(d v)}
$$

is equivalent to

$$
\sup _{\varepsilon>0}\left\|f A_{\varepsilon} \omega\right\|_{L^{1}(d \mu)} \leq C\|f \omega\|_{L^{1}(d \mu)}
$$

and this holds for all $f \in L^{1}(d v)$ if and only if

$$
M \omega(x)=\sup _{\varepsilon>0} A_{\varepsilon} \omega(x) \leq C \omega(x) \quad \text { a.e. }
$$


This means that the functions $\omega^{x}: \boldsymbol{R} \rightarrow \boldsymbol{R}, \omega^{x}(t)=\omega\left(\tau_{t} x\right)$, satisfy $A_{1}$ for almost every $x \in X$ with the same constant. Now we will use transference arguments to establish the weak type $(1,1)$ inequality for $H^{*}$. The proof for $M$ follows the same pattern and we omit it.

For fixed $\eta>0$ we define the truncated maximal ergodic Hilbert transform

$$
H_{\eta}^{*} f(x)=\sup _{\varepsilon>\eta}\left|\int_{\varepsilon<|t|<1 / \varepsilon} \frac{f\left(\tau_{t} x\right)}{t} d t\right| .
$$

Then, for all $N>0$ we have

$$
\begin{aligned}
v\left(\left\{x \in X: H_{\eta}^{*} f(x)>\lambda\right\}\right) & =\frac{1}{N} \int_{0}^{N} \int_{X} \chi_{\left\{x: H_{\eta}^{*} f(x)>\lambda\right\}}\left(\tau_{t} x\right) \omega\left(\tau_{t} x\right) d \mu(x) d t \\
& =\frac{1}{N} \int_{0}^{N} \int_{\left\{x \in X: H_{\eta}^{*} f\left(\tau_{t} x\right)>\lambda\right\}} \omega\left(\tau_{t} x\right) d \mu(x) d t .
\end{aligned}
$$

Since $H_{\eta}^{*} f\left(\tau_{t} x\right) \leq K^{*}\left(f^{x} \chi_{(-1 / \eta, N+1 / \eta)}\right)(t)$ and $\omega^{x}$ satisfies $A_{1}$ for almost all $x$ (with the same constant), Theorem B implies that

$$
\begin{aligned}
v\left(\left\{x \in X: H_{\eta}^{*} f(x)>\lambda\right\}\right) & \leq \frac{1}{N} \int_{X} \int_{\left\{t: K^{*}\left(f^{x} \chi_{(-1 / \eta, N+1 / \eta)}\right)(t)>\lambda\right\}} \omega^{x}(t) d t d \mu \\
& \leq \frac{C}{N \lambda} \int_{X} \int_{-1 / \eta}^{N+1 / \eta} f\left(\tau_{t} x\right) \omega\left(\tau_{t} x\right) d t d \mu=C \frac{N+2 / \eta}{N \lambda} \int_{X} f \omega d \mu
\end{aligned}
$$

because the flow preserves the measure $\mu$. Letting $N \rightarrow \infty$ and then $\eta \rightarrow 0$ we have the weak type $(1,1)$ inequality for $H^{*}$.

Now let $1<p<\infty$. The proof of the strong type inequalities will follow the same pattern of the above case, once we prove that, for almost every $x \in X$, the functions $\omega^{x}$ satisfy $A_{p}$ with the same constant. This can be proved as in [MT] and [S] where the discrete case was treated in a more general setting. We shall omit the details of the proof of the fact that $\omega^{x}$ satisfies $A_{p}$ and the transference argument.

The convergence of the averages $A_{\varepsilon} f$ and of $H_{\varepsilon} f$ follows from the weak type inequalities and the fact that the convergence holds for function $f \in L^{1}(d \mu) \cap L^{p}(\omega d \mu)$ which is dense in $L^{p}(\omega d \mu)$.

REMARK. It is worth noting that we only shall use the case $p>1$ for flows which are Cesàro bounded in $L^{1}(d v)$, i.e., for flows such that $\omega^{x}$ satisfies $A_{1}$ for almost every $x \in X$ with the same constant $C$. For these flows the condition $A_{p}, p>1$, follows from $A_{1}$ very easily and therefore the proof of the strong type $(p, p)$ inequality is easier than in the general case stated in Theorem 1 .

4. Some basic facts for the proof of Theorem 2. Assume that $(X, \mathscr{M}, v)$ is a finite 
measure space and $\left\{\tau_{t}: t \in \boldsymbol{R}\right\}$ is a nonsingular measurable fiow on $X$ such that

$$
\sup _{\varepsilon>0}\left\|A_{\varepsilon} f\right\|_{L^{1}(d v)} \leq C\|f\|_{L^{1}(d v)}
$$

for some constant $C$ and all functions $f \in L^{1}(d v)$. Then, as we have seen in Section 3, there exists a finite measure $\mu$ equivalent to $v$ such that the flow preserves $\mu$. If $\omega=d v / d \mu$ we know that the functions $\omega^{x}$ satisfy $A_{1}$ for almost every $x \in X$ with the same constant C.

Let $f$ be a measurable function on $X$ and let $\varphi: \boldsymbol{R} \rightarrow \boldsymbol{R}$ be a measurable function. The convolution of $f$ and $\varphi$ is defined by

$$
f * \varphi(x)=\int_{-\infty}^{\infty} f\left(\tau_{t} x\right) \varphi(t) d t
$$

if it makes sense (the convolution of functions in $\boldsymbol{R}$ will be denoted by the same symbol *). Since the flow preserves the measure $\mu$ it is very easy to see that if $f \in L^{1}(d \mu)$ and $\varphi \in L^{1}(\boldsymbol{R})$ then $f * \varphi$ is defined and

$$
\|f * \varphi\|_{L^{1}(d \mu)} \leq\|f\|_{L^{1}(d \mu)}\|\varphi\|_{L^{1}(\boldsymbol{R})} .
$$

We wish to show now that the convolution $f * \varphi$ is also defined for functions $f \in L^{1}(d v)$ and $\varphi \in L^{1}(\boldsymbol{R})$. In order to prove it, we recall that the function $\omega$ belongs to $L^{1}(d \mu)$ and satisfies the following:

$$
A_{\varepsilon} \omega(x) \leq C \omega(x) \quad \text { for all } \quad \varepsilon>0 \text { and almost every } \quad x \in X .
$$

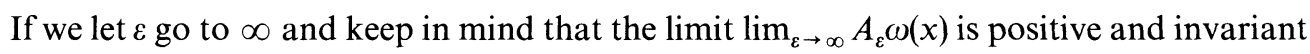
a.e. then we obtain

$$
0<\lim _{\varepsilon \rightarrow \infty} A_{\varepsilon} \omega(x) \leq C \inf _{t \in \mathbf{R}} \omega\left(\tau_{t} x\right) \quad \text { a.e. }
$$

Therefore if we consider for each integer $n$ the set

$$
X_{n}=\left\{x \in X: 2^{n} \leq \sup _{t \in R} \omega^{-1}\left(\tau_{t} x\right)<2^{n+1}\right\}
$$

we observe that the sets $X_{n}$ are invariant $\left(\tau_{t} X_{n}=X_{n}\right.$ for all $\left.t \in \boldsymbol{R}\right)$, pairwise disjoint and $X=\bigcup_{n} X_{n}$ (these equalities are understood up to a set of measure zero). Furthermore, $L^{1}\left(X_{n}, d v\right) \subset L^{1}\left(X_{n}, d \mu\right)$ because

$$
\int_{X_{n}}|f| d \mu=\int_{X_{n}}|f| \frac{1}{\omega} d v \leq 2^{n+1} \int_{X_{n}}|f| d v .
$$

Thus if $f \in L^{1}(d v)$ and $\varphi \in L^{1}(\boldsymbol{R})$ we have that the convolution $f * \varphi$ is defined on each $X_{n}$, since $f \in L^{1}\left(X_{n}, d v\right) \subset L^{1}\left(X_{n}, d \mu\right)$. Keeping in mind that the sets $X_{n}$ are invariant, pairwise disjoint and $X=\bigcup_{n} X_{n}$, we conclude that $f * \varphi$ is defined on $X$. 
This decomposition of the set $X$ allows to obtain more properties of the convolution which are necessary for the proof of Theorem 2. More precisely:

(a) If $f \in L^{1}(d v), H f \in L^{1}(d v)$ and $\varphi \in L^{1}(R)$ then $H(f * \varphi)=H f * \varphi$.

(b) If $f \in L^{1}(d v), \varphi \in L^{1}(\boldsymbol{R})$ and $K \varphi \in L^{1}(\boldsymbol{R})$ then $H(f * \varphi)=f * K \varphi$.

(Observe that $H(f * \varphi)$ is defined since $f * \varphi \in L^{1}\left(X_{n}\right)$ for each $n$ ). As before, these facts can be proved by reducing them to the case of the sets $X_{n}$ and the functions in $L^{1}\left(X_{n}, d \mu\right)$. This case, the measure preserving case, was proved in [ABG] in a more general setting.

We would like to obtain also an inequality like

$$
\|f * \varphi\|_{L^{1}(d \mu)} \leq\|f\|_{L^{1}(d \mu)}\|\varphi\|_{L^{1}(\boldsymbol{R})}
$$

but with the measure $v$. In the next theorem we give a result of this type under some assumptions on $\varphi$.

THEOREM 3. Let $(X, \mathscr{M}, v)$ be a finite measure space and let $\left\{\tau_{t}: t \in \boldsymbol{R}\right\}$ be a nonsingular measurable flow on $X$ such that

$$
\sup _{\varepsilon>0}\left\|A_{\varepsilon} f\right\|_{L^{1}(d v)} \leq C\|f\|_{L^{1}(d v)}
$$

for some positive constant $C$ and all functions $f \in L^{1}(d v)$. Assume that $\varphi \in L^{1}(\boldsymbol{R})$ and $\psi(t)=\sup _{|s| \geq|t|}|\varphi(s)|$ is in $L^{1}(\boldsymbol{R})$ ( $\psi$ is the least radial decreasing majorant of $\left.\varphi\right)$. If $f \in L^{1}(d v)$ then

$$
|f * \varphi(x)| \leq\|\psi\|_{L^{1}(\boldsymbol{R})} M f(x)
$$

and

$$
\|f * \varphi\|_{L^{1}(d v)} \leq C\|\psi\|_{L^{1}(\boldsymbol{R})}\|f\|_{L^{1}(d v)}
$$

where the constant $C$ is independent of $f$ and $\varphi$.

Proof. For fixed $x \in X$ let us denote by $f^{x}$ the function $f^{x}(s)=f\left(\tau_{s} x\right)$. Then $|f * \varphi(x)| \leq|f| *|\varphi|(x) \leq\left|f^{x}\right| * \varphi(0)$ where the last $*$ stands for the convolution of functions in $\boldsymbol{R}$. By a standard result (see [GC] for instance) the last term is dominated by $\|\psi\|_{1}\left(f^{x}\right)^{*}(0)$. But $\left(f^{x}\right)^{*}(0)=M f(x)$. This proves the first inequality.

Let $\mu$ and $\omega$ be as above. By Fubini's theorem and the fact that the flow preserves the measure $\mu$ we obtain

$$
\|f * \varphi\|_{L^{1}(d v)} \leq \int_{X}|f(x)|\left(\int_{-\infty}^{\infty} \omega\left(\tau_{s} x\right)|\varphi(-s)| d s\right) d \mu=\int_{X}|f(x)| \omega *|\bar{\varphi}|(x) d \mu
$$

where $\bar{\varphi}(s)=\varphi(-s)$. Then by what we have already shown

$$
\|f * \varphi\|_{L^{1}(d v)} \leq\|\psi\|_{L^{1}(\boldsymbol{R})} \int_{X}|f(x)| M \omega(x) d \mu .
$$


Now, since the functions $\omega^{x}$ satisfy $A_{1}$ for almost every $x \in X$ with the same constant, we have

$$
\|f * \varphi\|_{L^{1}(d v)} \leq C\|\psi\|_{L^{1}(\boldsymbol{R})} \int_{X}|f| \omega d \mu .
$$

The proof of Theorem 2 requires to approximate the functions in $L^{1}(d v)$ by suitable functions. This is the next result.

TheOREM 4. Let $(X, \mathscr{M}, v)$ and $\left\{\tau_{t}: t \in \boldsymbol{R}\right\}$ be as in Theorem 3. Let

$$
A=\left\{f \in L^{1}(d v): f(x)=f\left(\tau_{t} x\right) \text { a.e. } x \text { for all } t \in \boldsymbol{R}\right\}
$$

and

$$
B=\left\{f=g * \phi: g \in L^{\infty}(d v), \phi \text { bounded with compact support and } \int_{-\infty}^{\infty} \phi(s) d s=0\right\} .
$$

If $\bar{B}$ is the $L^{1}(d v)$-closure of the linear manifold generated by $B$ then $A \oplus \bar{B}=L^{1}(d v)$, i.e., $A \cap \bar{B}=\{0\}$ and $L^{1}(d v)=\left\{f_{1}+f_{2}: f_{1} \in A, f_{2} \in \bar{B}\right\}$.

Proof. It is clear that $A$ and $B$ are contained in $L^{1}(d v)$. We also have that $\lim _{\varepsilon \rightarrow \infty} A_{\varepsilon} f=f$ for all $f \in A$ and $\lim _{\varepsilon \rightarrow \infty} A_{\varepsilon} f=0$ if $f \in \bar{B}$ (these two facts give immediately that $A \cap B=\{0\}$ ). We only have to show the statement for the functions $f \in \bar{B}$. By the assumption

$$
\sup _{\varepsilon>0}\left\|A_{\varepsilon} f\right\|_{L^{1}(d v)} \leq C\|f\|_{L^{1}(d v)}
$$

it suffices to establish that $\lim _{\varepsilon \rightarrow \infty} A_{\varepsilon} f=0$ for all functions $f \in B$. In order to see this, we consider $f=g * \phi, g \in L^{\infty}(d v), \phi$ bounded with compact support and $\int_{-\infty}^{\infty} \phi(s) d s=0$. Then

$$
\begin{aligned}
A_{\varepsilon} f(x) & =\frac{1}{2 \varepsilon} \int_{-\varepsilon}^{\varepsilon} \int_{-\infty}^{\infty} g\left(\tau_{t+s} x\right) \phi(s) d s d t=\frac{1}{2 \varepsilon} \int_{-\infty}^{\infty} \phi(s) \int_{-\varepsilon}^{\varepsilon} g\left(\tau_{t+s} x\right) d t d s \\
& =\int_{-\infty}^{\infty} \phi(s) \frac{1}{2 \varepsilon}\left[\int_{-\varepsilon}^{\varepsilon}\left(g\left(\tau_{t+s} x\right)-g\left(\tau_{t} x\right)\right) s t\right] d s .
\end{aligned}
$$

By the dominated convergence theorem and the fact that

$$
\lim _{\varepsilon \rightarrow \infty} \frac{1}{2 \varepsilon} \int_{-\varepsilon}^{\varepsilon} g\left(\tau_{t+s} x\right) d t=\lim _{\varepsilon \rightarrow \infty} \frac{1}{2 \varepsilon} \int_{-\varepsilon}^{\varepsilon} g\left(\tau_{t} x\right) d t
$$

we obtain $\lim _{\varepsilon \rightarrow \infty} A_{\varepsilon} f(x)=0$.

Now we are going to prove that $A \oplus \bar{B}=L^{1}(d v)$. Let $f \in L^{1}(d v)$ and denote $F=\lim _{\varepsilon \rightarrow \infty} A_{\varepsilon} f$. It is clear that $F \in A$. Then it suffices to show that $f-F \in \bar{B}$. Assume that $h \in L^{\infty}(d v)$ and 


$$
\int_{X} h G d v=0 \quad \text { for all functions } \quad G \in \bar{B}
$$

Then

$$
\int_{X} h(x)\left(\int_{-\infty}^{\infty} g\left(\tau_{s} x\right) \phi(s) d s\right) \omega(x) d \mu=0
$$

for all functions $g \in L^{\infty}(d v)$ and all $\phi$ bounded with compact support and $\int_{-\infty}^{\infty} \phi(s) d s=0$. Then it follows from Theorem 3 that the above equality holds for all functions $g \in L^{1}(d v)$. Applying this equality to $g=f$ and $\phi=(1 / 2 \varepsilon) \chi_{(-\varepsilon, \varepsilon)}-(1 / 2 \eta) \chi_{(-\eta, \eta)}$ we obtain

$$
\int_{X} h(x) \omega(x) \frac{1}{2 \varepsilon} \int_{-\varepsilon}^{\varepsilon} f\left(\tau_{s} x\right) d s d \mu=\int_{X} h(x) \omega(x) \frac{1}{2 \eta} \int_{-\eta}^{\eta} f\left(\tau_{s} x\right) d s d \mu .
$$

Since $(1 / 2 \eta) \int_{-\eta}^{\eta} f\left(\tau_{s} x\right) d s$ converges to $F$ in the $L^{1}(d v)$-norm as $\eta$ goes to $\infty$ (Corollary 1) and $(1 / 2 \varepsilon) \int_{-\varepsilon}^{\varepsilon} f\left(\tau_{s} x\right) d s$ converges to $f$ in the $L^{1}(d v)$-norm as $\varepsilon$ goes to $0^{+}$(by Wiener's theorem and $\left.\sup _{\varepsilon>0}\left\|A_{\varepsilon} f\right\|_{L^{1}(d v)} \leq C\|f\|_{L^{1}(d v)}\right)$, we obtain

$$
\int_{X} f h \omega d \mu=\int_{X} F h \omega d \mu \text {, i.e., } \int_{X}(f-F) h d v=0
$$

for the functions $h \in L^{\infty}(d v)$ as above. This means that $f-F \in \bar{B}$.

In order to finish this section we are going to study the behaviour of the convolutions $f * \varphi_{\varepsilon}$ where $\varphi$ is continuous with compact support and $\varphi_{\varepsilon}(t)=(1 / \varepsilon) \varphi(t / \varepsilon)$.

Theorem 5. Let $(X, \mathscr{M}, v),\left\{\tau_{t}: t \in \boldsymbol{R}\right\}, A, B$ and $\bar{B}$ be as in Theorem 4. Let $\varphi: \boldsymbol{R} \rightarrow \boldsymbol{R}$ be continuous with compact support.

(a) If $f \in L^{1}(d v)$ then $f * \varphi_{\varepsilon}$ converges to $\left(\int_{-\infty}^{\infty} \varphi(s) d s\right) f$ in the $L^{1}(d v)$-norm as $\varepsilon$ goes to 0 .

(b) If $f \in \bar{B}$ then $f * \varphi_{1 / \varepsilon}$ converges to 0 in the $L^{1}(d v)$-norm as $\varepsilon$ goes to 0 .

Proof. Let $\mu$ and $w$ be as in the proof of Theorem 4. By Theorem 4 and since

$$
\sup _{\varepsilon>0}\left\|A_{\varepsilon} f\right\|_{L^{1}(d v)} \leq C\|f\|_{L^{1}(d v)},
$$

it suffices to establish the results for functions $f \in A \cup B$. If $f \in A$ it is clear that $f * \varphi_{\varepsilon}(x)=f(x) \int_{-\infty}^{\infty} \varphi(s) d s$ and therefore (a) follows for functions $f \in A$. Now let $f \in B$. Then $f=g * \phi$ where $g \in L^{\infty}(d v), \phi$ is bounded with compact support and $\int_{-\infty}^{\infty} \phi(s) d s=0$. In what follows if $\psi: \boldsymbol{R} \rightarrow \boldsymbol{R}$, we write $\bar{\psi}$ to denote the function $\bar{\psi}(s)=\psi(-s)$. Then by Fubini's theorem and the fact that the flow preserves the measure $\mu$ we obtain

$$
\left\|f * \varphi_{\varepsilon}-f \int_{-\infty}^{\infty} \varphi(s) d s\right\|_{L^{1}(d v)}=\left\|g *\left(\phi * \varphi_{\varepsilon}\right)-(g * \phi) \int_{-\infty}^{\infty} \varphi(s) d s\right\|_{L^{1}(d v)}
$$




$$
\begin{aligned}
& \leq \int_{X}|g| \overline{\left(\phi * \varphi_{\varepsilon}-\phi \int_{-\infty}^{\infty} \varphi(s) d s\right)} * \omega d \mu \leq\|g\|_{\infty}\left\|\overline{\left(\phi * \varphi_{\varepsilon}-\phi \int_{-\infty}^{\infty} \varphi(s) d s\right)} * \omega\right\|_{L^{1}(d \mu)} \\
& \leq\|g\|_{\infty}\left\|\phi * \varphi_{\varepsilon}-\phi \int_{-\infty}^{\infty} \varphi(s) d s\right\|_{L^{1}(\boldsymbol{R})} v(X) .
\end{aligned}
$$

By elementary results in the real line,

$$
\lim _{\varepsilon \rightarrow 0^{+}}\left\|\phi * \varphi_{\varepsilon}-\phi \int_{-\infty}^{\infty} \varphi(s) d s\right\|_{L^{1}(\boldsymbol{R})}=0
$$

and therefore (a) is proved.

Now we shall prove (b). As before it suffices to prove it for functions $f \in B$. Let $f=g * \phi$ with $g \in L^{\infty}(d v), \phi: \boldsymbol{R} \rightarrow \boldsymbol{R}$ bounded with compact support and $\int_{-\infty}^{\infty} \phi(s) d s=0$. Since $\phi * \varphi_{1 / \varepsilon}=\left(\phi_{\varepsilon} * \varphi\right)_{1 / \varepsilon}$ we have

$$
\left\|f * \varphi_{1 / \varepsilon}\right\|_{L^{1}(d v)} \leq \int_{X}|g| *\left|\left(\phi_{\varepsilon} * \varphi\right)_{1 / \varepsilon}\right| \omega d \mu .
$$

By Fubini's theorem and the fact that the flow preserves the measure $\mu$ we have

$$
\begin{aligned}
\left\|f * \varphi_{1 / \varepsilon}\right\|_{L^{1}(d v)} & \leq \int_{X}|g| \overline{\left(\phi_{\varepsilon} * \varphi\right)_{1 / \varepsilon}} * \omega d \mu \leq\|g\|_{\infty}\left\|\overline{\left(\phi_{\varepsilon} * \varphi\right)_{1 / \varepsilon}} * \omega\right\|_{L^{1}(d \mu)} \\
& \leq\|g\|_{\infty}\left\|\overline{\left(\phi_{\varepsilon} * \varphi\right)_{1 / \varepsilon}}\right\|_{L^{1}(\boldsymbol{R})} v(X) \leq\|g\|_{\infty} v(X)\left\|\phi_{\varepsilon} * \varphi\right\|_{L^{1}(\boldsymbol{R})} .
\end{aligned}
$$

Again, by elementary results $\phi_{\varepsilon} * \varphi$ converges to $\varphi \int_{-\infty}^{\infty} \phi(s) d s=0$ in $L^{1}(\boldsymbol{R})$. Therefore $\lim _{\varepsilon \rightarrow 0^{+}}\left\|f * \varphi_{1 / \varepsilon}\right\|_{L^{1}(d v)}=0$.

5. Proof of Theorem 2. Let $\mu, \omega, A, B$ and $\bar{B}$ be as in Section 4. Let $f \in L^{1}(d v)$ such that $H f \in L^{1}(d v)$. Then $f=f_{1}+f_{2}, f_{1} \in A$ and $f_{2} \in \bar{B}$. Since $H_{\varepsilon} f_{1}=H f_{1}=0$ it is clear that we may assume that $f \in \bar{B}$.

Let $f \in \bar{B}$ such that $H f \in L^{1}(d v)$. Consider a function $\varphi: \boldsymbol{R} \rightarrow \boldsymbol{R}, \varphi>0$ continuous with compact support and $\int_{-\infty}^{\infty} \varphi(t) d t=1$. Let $k(x)=1 / x$ if $|x|>1$ and $k(x)=0$ if $|x| \leq 1$. Finally let $\delta=K \varphi-k$ and $h_{\varepsilon}=k_{\varepsilon}-k_{1 / \varepsilon}$. Observe that $H_{\varepsilon} f=f * h_{\varepsilon}$ and since the dilation commutes with the Hilbert transform we get

$$
h_{\varepsilon}=K \varphi_{\varepsilon}-K \varphi_{1 / \varepsilon}+\delta_{1 / \varepsilon}-\delta_{\varepsilon} .
$$

Following the ideas in [CS] we see that the least radial decreasing majorant of $\delta$ is in $L^{1}(\boldsymbol{R})$ and therefore for $\Delta_{\varepsilon}=\delta_{1 / \varepsilon}-\delta_{\varepsilon}$ we get (Theorem 3)

$$
\left\|F * \Delta_{\varepsilon}\right\|_{L^{1}(d v)} \leq C\|F\|_{L^{1}(d v)} \quad \text { for all } \quad F \in L^{1}(d v) .
$$

Let $\gamma>0$ and choose $g$ in the linear manifold generated by $B$ and such that $\|f-g\|_{L^{1}(d v)} \leq \gamma$. Then 


$$
\left\|H_{\varepsilon} f-H f\right\|_{L^{1}(d v)}=\left\|f * h_{\varepsilon}-H f\right\|_{L^{1}(d v)}=\left\|f * \Delta_{\varepsilon}-f * K\left(\varphi_{1 / \varepsilon}-\varphi_{\varepsilon}\right)-H f\right\|_{L^{1}(d v)} .
$$

Since $K\left(\varphi_{1 / \varepsilon}-\varphi_{\varepsilon}\right) \in L^{1}(\boldsymbol{R}), \varphi_{1 / \varepsilon}-\varphi_{\varepsilon} \in L^{1}(\boldsymbol{R})$ and $H f \in L^{1}(d v)$, the properties (a) and (b) in Section 4 give

$$
\begin{aligned}
\left\|H_{\varepsilon} f-H f\right\|_{L^{1}(d v)}= & \left\|f * \Delta_{\varepsilon}-H f * \varphi_{1 / \varepsilon}+H f * \varphi_{\varepsilon}-H f\right\|_{L^{1}(d v)} \\
\leq & \left\|H f * \varphi_{\varepsilon}-H f\right\|_{L^{1}(d v)}+\left\|(f-g) * \Delta_{\varepsilon}\right\|_{L^{1}(d v)} \\
& +\left\|g * \Delta_{\varepsilon}\right\|_{L^{1}(d v)}+\left\|H f * \varphi_{1 / \varepsilon}\right\|_{L^{1}(d v)} .
\end{aligned}
$$

Now, since $K\left(\varphi_{\varepsilon}-\varphi_{1 / \varepsilon}\right) \in L^{1}(\boldsymbol{R})$ and $H g \in L^{1}(d v)$,

$$
\begin{aligned}
g * \Delta_{\varepsilon} & =H_{\varepsilon} g-g * K\left(\varphi_{\varepsilon}-\varphi_{1 / \varepsilon}\right)=H_{\varepsilon} g-H g *\left(\varphi_{\varepsilon}-\varphi_{1 / \varepsilon}\right) \\
& =H_{\varepsilon} g-H g+H g-H g *\left(\varphi_{\varepsilon}-\varphi_{1 / \varepsilon}\right) .
\end{aligned}
$$

Therefore

$$
\begin{aligned}
\left\|H_{\varepsilon} f-H f\right\|_{L^{1}(d v)} \leq & \left\|H f * \varphi_{\varepsilon}-H f\right\|_{L^{1}(d v)}+\left\|(f-g) * \Delta_{\varepsilon}\right\|_{L^{1}(d v)} \\
& +\left\|H_{\varepsilon} g-H g\right\|_{L^{1}(d v)}+\left\|H g-H g * \varphi_{\varepsilon}\right\|_{L^{1}(d v)} \\
& +\left\|H g * \varphi_{1 / \varepsilon}\right\|_{L^{1}(d v)}+\left\|H f * \varphi_{1 / \varepsilon}\right\|_{L^{1}(d v)} .
\end{aligned}
$$

Since $H f \in L^{1}(d v)$, Theorem 5 gives that $\left\|H f * \varphi_{\varepsilon}-H f\right\|_{L^{1}(d v)}+\left\|H g-H g * \varphi_{\varepsilon}\right\|_{L^{1}(d v)}$ tends to 0 as $\varepsilon$ goes to 0 . By one of the above inequalities

$$
\left\|(f-g) * \Delta_{\varepsilon}\right\|_{L^{1}(d v)} \leq C\|f-g\|_{L^{1}(d v)} \leq C \gamma .
$$

By Corollary 1 and the fact that the linear manifold generated by $B$ is contained in $L^{2}(d v)$ we have that $\left\|H_{\varepsilon} g-H g\right\|_{L^{2}(d v)} \rightarrow 0$ as $\varepsilon$ goes to 0 and therefore $\lim _{\varepsilon \rightarrow 0^{+}}\left\|H_{\varepsilon} g-H g\right\|_{L^{1}(d v)}=0$ because $v(X)<\infty$. Finally, by Theorem 5,

$$
\lim _{\varepsilon \rightarrow 0^{+}}\left(\left\|H f * \varphi_{1 / \varepsilon}\right\|_{L^{1}(d v)}+\left\|H g * \varphi_{1 / \varepsilon}\right\|_{L^{1}(d v)}\right)=0
$$

if we establish that $H f$ and $H g$ belong to $\bar{B}$. This is proved in the next theorem (observe that $H f \in L^{1}(d v)$ and $\left.H g \in L^{1}(d v)\right)$.

THEOREM 6. Under the assumptions of Theorem 2, if $f \in L^{1}(d v)$ and $H f \in L^{1}(d v)$ then $H f \in \bar{B}$.

Proof. Consider as in Section 4 the sets

$$
X_{n}=\left\{x \in X: 2^{n} \leq \sup _{t \in \mathbb{R}} \omega^{-1}\left(\tau_{t} x\right)<2^{n+1}\right\} .
$$

Let $f \in L^{1}(d v)$ such that $H f \in L^{1}(d v)$. Since $L^{1}\left(X_{n}, d v\right) \subset L^{1}\left(X_{n}, d \mu\right)$ then $f \in L^{1}\left(X_{n}, d \mu\right)$ and $H f \in L^{1}\left(X_{n}, d \mu\right)$. Therefore, by Theorem (3.23) in [ABG] we have that for each $n$ there exist $F_{n} \in L^{1}\left(X_{n}, d \mu\right)$ and $\phi_{n}: \boldsymbol{R} \rightarrow \boldsymbol{R}$ bounded with compact support, $\int_{-\infty}^{\infty} \phi_{n}(s) d s=0$, and 
such that $H F_{n} \in L^{1}\left(X_{n}, d \mu\right)$ and $f=F_{n} * \phi_{n}$ on $X_{n}$. As a consequence of the properties (a) and (b) in Section 4

$$
H f=H F_{n} * \phi_{n} \quad \text { on } \quad X_{n} .
$$

On the other hand, since $H f \in L^{1}(d v)$ we have by Theorem 4 that

$$
H f=f_{1}+f_{2}, \quad f_{1} \in A, \quad f_{2} \in \bar{B} .
$$

Now we observe that

$$
\lim _{\varepsilon \rightarrow \infty} A_{\varepsilon}(H f)=\lim _{\varepsilon \rightarrow \infty} A_{\varepsilon}\left(H F_{n} * \phi_{n}\right)=0 \quad \text { on } \quad X_{n}
$$

and

$$
\lim _{\varepsilon \rightarrow \infty} A_{\varepsilon}(H f)=\lim _{\varepsilon \rightarrow \infty}\left(A_{\varepsilon} f_{1}+A_{\varepsilon} f_{2}\right)=\lim _{\varepsilon \rightarrow \infty} A_{\varepsilon} f_{1}=f_{1} .
$$

Therefore $f_{1}=0$ and $H f=f_{1}+f_{2}=f_{2} \in \bar{B}$.

6. Examples of Cesàro bounded flows. This section is devoted to providing examples of Cesàro bounded flows.

We begin by considering the case $X=\{z \in C:|z|=1\}$ with the $\sigma$-algebra of the Borel sets and the flow $\tau_{t}(z)=z e^{i t}$. It follows from Theorem 1 and its proof that $v$ is a measure such that the flow is Cesàro bounded in $L^{p}(d v)$ if and only if $d v=\omega d \mu$ where the flow preserves the measure $\mu, \omega>0$ a.e. and $\omega^{z}$ satisfies $A_{p}$ for almost all $z \in X$. In our case this means that $d v\left(e^{i t}\right)=\omega\left(e^{i t}\right) d t$ for some $\omega>0$ a.e. which satisfies Muckenhoupt's $A_{p}$ condition. These weights $\omega$ were studied in $[\mathrm{Mu}]$ and $[\mathrm{HMuW}]$ (see also $[\mathrm{GR}]$ ). For instance, we have that $\omega\left(e^{i t}\right)=t^{\alpha},|t| \leq \pi$, is an $A_{p}$ weight if and only if $-1<\alpha<p-1$ and in this case the flow is Cesàro bounded in $L^{p}(d v)$ if $d v\left(e^{i t}\right)=\omega\left(e^{i t}\right) d t$. More examples can be given in this setting by using the result of Coifmann and Rochberg [CR] and the factorization of $A_{p}$ weights (see [GR]).

Next, we are going to show nontrivial examples in a more general setting.

THEOREM 7. Let $(X, \mathscr{M}, \mu)$ be a nonatomic finite measure space and let $\left\{\tau_{t}: t \in \boldsymbol{R}\right\}$ be a measure preserving flow such that $\tau_{\boldsymbol{t}}$ is ergodic for some $t \in \boldsymbol{R}$. Then for every $p \geq 1$ and every $q, 1 \leq q<p$, there exists a measure $d v=\omega d \mu, \omega>0$ a.e., such that the following holds:

(1) The flow is Cesàro bounded in $L^{p}(d v)$ and is not Cesàro bounded in $L^{q}(d v)$.

(2) The flow is not power bounded in $L^{p}(d v)$.

Proof. We begin by proving the case $p=1$. Let $f>0, f \in L^{2}(d \mu), f \notin L^{\infty}(d \mu)$. Denote by $M^{(i)}$ the iterations of the ergodic maximal operator associated to the flow. We know that $M$ is bounded in $L^{2}(d \mu)$, more precisely,

$$
\|M f\|_{L^{2}(d \mu)} \leq 2\|f\|_{L^{2}(d \mu)} .
$$


Let

$$
\omega=\sum_{i=0}^{\infty} \frac{1}{4^{i}} M^{(i)} f
$$

Then $\omega \geq f>0, \omega \in L^{2}(d \mu)$ and

$$
M \omega \leq \sum_{i=0}^{\infty} \frac{1}{4^{i}} M^{(i+1)} f \leq 4 \omega .
$$

Therefore, $w^{x}$ satisfies $A_{1}$. Then it is clear that (1) holds for $d v=\omega d \mu$ and $p=1$. Let us see now that (2) does not hold. Assume that the flow is power bounded in $L^{1}(d v)$. This implies immediately that there exists a constant $C>0$ such that

$$
\omega\left(\tau_{t} x\right) \leq C \omega(x) \text { a.e. }
$$

for all $t \in \boldsymbol{R}$. Then the assumption about the ergodicity of the flow gives that $\omega \in L^{\infty}(d \mu)$ which is a contradiction since $0<f \leq \omega$ and $f \notin L^{\infty}(d \mu)$.

Let us consider now the case $p>1$. Let $1<r<(p-1) /(q-1)$ and let $A$ be a positive constant such that

$$
\|M f\|_{L^{r}(d \mu)} \leq A\|f\|_{L^{r}(d \mu)}
$$

for all $f \in L^{r}(d \mu)$. Now choose $f \in L^{r}(d \mu)$ such that $f \notin L^{(p-1) /(q-1)}(d \mu)$ and set

$$
\omega=\sum_{i=0}^{\infty} \frac{1}{A^{2 i}} M^{(i)} f .
$$

As before, $\omega^{x}$ satisfies $A_{1}$. Then Hölder's inequality shows that if $u=\omega^{1-p}$ then $u^{x}$ satisfies $A_{p}$ and therefore the flow is Cesàro bounded in $L^{p}(d v), d v=u d \mu$. In order to prove the statement (1) completely, we assume that the flow is Cesàro bounded in $L^{q}(d v)$, i.e., $u^{x}$ satisfies $A_{q}$ with the same constant for almost all $x \in X$. This implies

$$
A_{N} u(x)\left(A_{N} u^{-1 /(q-1)}(x)\right)^{q-1} \leq C
$$

for some constant $C$. Letting $N$ go to $\infty$ and keeping in mind that $\tau_{t}$ is ergodic for some $t$, we get that $u^{-1 /(q-1)} \in L^{1}(d \mu)$, i.e., $\omega \in L^{(p-1) /(q-1)}(d \mu)$. This is a contradiction to the fact that $w \geq f \notin L^{(p-1) /(q-1)}$.

Finally, observe that (2) follows from (1) since (2) implies that there exists $C>0$ such that for all $t$

$$
u\left(\tau_{t} x\right) \leq C u(x) \text { a.e. }
$$

and this would immediately imply that the flow is Cesàro bounded in $L^{1}(d v)$ and then in $L^{r}(d v)$ for all $r, 1 \leq r<\infty$.

REMARK. The theorem and its proof follow the ideas of the theory of weights (see $[\mathrm{CJR}]$ and $[\mathrm{GR}]$ ). 


\section{REFERENCES}

[ABG] N. Asmar, E. Berkson ANd T. A. Gillespie, Distributional control and generalized analitycity, Integral Equations and Operator Theory 14 (1991), 311-340.

[CC] A. P. Calderón, O. N. CaPRi and C. Segovia, On the convergence in $L^{1}$ of singular integrals, Studia Math. 78 (1984), 321-327.

[CS] O. N. CAPRI AND C. SEgovia, Convergence of singular integrals in weighted $L^{1}$ spaces, Trabajos de Matemática 99, Instituto Argentino de Matemática (IAM-CONICET), Buenos Aires (1986).

[CF] R. R. CoIfMAN AND C. FEFFERman, Weighted norm inequalities for maximal functions and singular integrals, Studia Math. 51 (1974), 241-250.

[CJR] R. Coifman, P. W. Jones and J. L. Rubio de Francia, Constructive decomposition of B.M.O., functions and factorization of $A_{p}$ weights, Proc. Amer. Math. Soc. 87 (1983), 675-676.

[CR] R. Coifman ANd R. Rochberg, Another characterization of B.M.O., Proc. Amer. Math. Soc. 79 (1980), 249-254.

[GR] J. García-Cuerva AND J. L. Rubio de Francia, Weighted norm inequalities and related topics, North-Holland, Amsterdam, New York, Oxford, 1985.

[HMuW] R. A. Hunt, B. Muckenhoupt AND R. L. WheEden, Weighted norm inequalities for the conjugate function and the Hilbert transform, Trans. Amer. Math. Soc. 176 (1973), 261-274.

[MT] F. J. MARTín-ReYes AND A. DE LA TORRE, The dominated ergodic estimate for mean bounded, invertible, positive operators, Proc. Amer. Math. Soc. 104 (1988), 69-75.

[Mu] B. Muckenhoupt, Weighted norm inequalities for the Hardy maximal function, Trans. Amer. Math. Soc. 165 (1972), 207-226.

[P] K. Petersen, Ergodic Theory, Cambridge Studies in Advanced Math. 2, Cambridge Univ. Press, 1983.

[S] R. SATo, A remark on the ergodic Hilbert transform, Math. J. Okayama Univ. 28 (1986), 159-163.

\begin{tabular}{|c|c|}
\hline ANÁlisis MATEMÁtico & ANÁlisis MATEMÁtico \\
\hline Facultad de Ciencias & Facultad de Ciencias \\
\hline UNIVERSIDAD DE MÁlaGA & UNIVERSIDAD DE MÁLAGA \\
\hline 29071 MÁlaGA & 29071 MÁLAGA \\
\hline SPAIN & SPAIN \\
\hline
\end{tabular}

